Titre : La santé publique et l'heuristique personnelle

Auteur : Noni E. MacDonald

Publication : La santé publique à une ère marquée par le doute - Origines religieuses et culturelles de l'hésitation des Canadiens face à la vaccination

Directeurs : Paul Bramadat, Maryse Guay, Julie A. Bettinger et Réal Roy

Pages : $314-318$

ISBN : 978-2-7622-0359-2

URI : http://hdl.handle.net/11143/16033

DOI : https://doi.org/10.17118/11143/16033

Éditeur : Les Éditions de l'Université de Sherbrooke (ÉDUS) 


\title{
La santé publique et l'heuristique personnelle
}

\author{
NONI E. MACDONALD
}

Comme Goethe l'écrivait en 1774 , la foi n'est pas le début de la connaissance, mais son aboutissement; selon lui, nous sommes constitués de telle façon que l'incroyable nous semble vrai et qu'une fois cette vérité imprimée dans notre mémoire, il est fort peu probable qu'on puisse la faire disparaître. Plusieurs des coauteurs de cet ouvrage laissent entendre que ce principe s'applique à ceux qui veulent remettre en question autant des croyances au sujet des vaccins et de la santé que des convictions religieuses.

Depuis que l'humanité existe, I'homme a à cœur d'entretenir sa santé. À toute heure du jour, les adultes prennent des décisions pour qu'eux-mêmes, leur famille et leur collectivité demeurent en santé : on se nourrit sainement, on dort suffisamment, on fait de l'exercice, on se lave les mains, on pratique l'hygiène personnelle, et ainsi de suite. Bien qu'au-delà de 80 p. cent des parents canadiens veillent à ce que leurs enfants reçoivent les vaccins recommandés, certains de leurs semblables, de même que des professionnels de la santé et des citoyens en général, demeurent indécis en matière de vaccination (Gust et coll., 2008; Salmon et coll., 2005). Un sondage de grande envergure mené en 2011 a permis de conclure que les enfants dans la vaste majorité des familles canadiennes avait une vaccination à jour; parmi celles dont les enfants n'étaient pas adéquatement vaccinés, 28 p. cent croyaient les vaccins inutiles, affirmant que le corps humain pouvait se protéger lui-même, 16 p. cent s'inquiétaient de l'innocuité des vaccins et autant les rejetaient pour des motifs religieux ou philosophiques?. Par ailleurs, $5 \mathrm{p}$. cent des répondants estimaient les vaccins pédiatriques inefficaces et 10 p. cent étaient indécis quant à leurs avantages. Bien que l'innocuité des vaccins soit un facteur important pour bien des parents réticents (Salmon et coll., 2005), on retrouve une même inquiétude chez certains travailleurs de la santé (Riphagen-Dalhuisen, Gefenaite et Hak, 2012).

1. Se reporter à «Sondage auprès des parents sur des questions clés en matière d'immunisation »: Rapport définitif, septembre 2011, http://resources.cpha.ca/immunize.ca/data/1795f.pdf 
Comment la réticence à la vaccination peut-elle se maintenir malgré la solidité des données scientifiques démontrant que les bienfaits des vaccins sont supérieurs à leurs risques, que les personnes non immunisées courent de grands risques en cas de maladie et que les complications d'une maladie contagieuse sont graves?

Les connaissances heuristiques - ces raccourcis cognitifs dont nous nous servons tous inconsciemment pour simplifier la prise d'une décision complexe - minent notre conviction que les vaccins sont nécessaires et sûrs (MacDonald, Smith et Appleton, 2012). L'heuristique de disponibilité (qui nous amène à juger de la fréquence ou de la probabilité d'un événement selon que nous pouvons facilement l'imaginer ou nous le rappeler), par exemple, amènera peut-être un parent à se souvenir aisément d'un reportage ou d'une anecdote racontée par un ami alléguant que l'autisme est attribuable aux vaccins, sans qu'il ait auparavant vu ou entendu parler d'un enfant ayant contracté la rougeole, la pneumonie ou une encéphalite. Selon la conviction qui s'enracine dans l'esprit de ce parent - parfois de manière inconsciente -, les vaccins sont dangereux et les maladies qu'ils doivent prévenir ne le sont pas. Les parents peuvent croire, en toute conscience, qu'ils hésitent encore, mais la décision a déjà été prise dans leur subconscient (Galdi, Arcuri et Gawronski, 2008).

La décision de refuser la vaccination peut aussi être influencée par Internet, par les médias sociaux et par les médias traditionnels (Galdi, Arcuri et Gawronski, 2008; Scullard, Peacock et Davies, 2010). II suffit de cinq à dix minutes de consultation d'un site Web anti-vaccination pour que les perceptions sur les vaccins et la volonté d'accepter la vaccination se modifient (Betsch et coll., 2010). Les blogues qui critiquent la vaccination ont aussi un effet très négatif (Nan et Madden, 2012). Malgré les erreurs de logique, la pensée magique et les distorsions de la réalité que l'on retrouve sur les sites anti-vaccination (Jacobson, Targonski et Poland, 2007), les histoires et les images d'enfants rendus malades par un vaccin sont si impressionnantes qu'elles restent imprimées dans l'esprit et peuvent avoir une influence négative sur la prise de décision des parents.

Si les preuves scientifiques suffisaient, personne ne fumerait, ni n'absorberait de malbouffe, tous feraient de l'exercice physique tous les jours et chacun serait entièrement immunisé. Malheureusement, l'esprit filtre l'information qui lui est présentée, de sorte qu'à la réception de nouvelles connaissances qui s'allient bien avec les convictions personnelles, il les sélectionne ou se les rappelle, justement à cause de ce renforcement. Si ces renseignements vont à l'encontre des convictions existantes, l'esprit aura tendance à les rejeter. Les parents, notamment, font appel à d'autres formes de raisonnement qui sont problématiques ou à tout le moins bizarres lorsqu'il s'agit de prendre des décisions de vaccination; bon nombre de ces approches sont analysées dans le présent ouvrage.

Vu l'importance d'une couverture vaccinale élevée pour la santé individuelle et collective, il est essentiel d'influencer positivement les croyances sur la vaccination autant que de réfuter les croyances négatives. Par contre, les croyances et les préoccupations, en matière de vaccination, varient grandement. Les auteurs de ces pages ont choisi de se pencher sur les racines culturelles et religieuses de la réticence à la vaccination au Canada. Ils donnent des arguments logiques pour remplacer la stratégie simpliste actuellement utilisée pour réfuter des interrogations sur la vaccination, soit de répliquer au moyen de données probantes. En comprenant mieux les origines de ces interrogations, les raisons pour lesquelles elles demeurent populaires et les efforts que font les cliniciens, les scientifiques et les défenseurs 
de la santé publique au Canada pour améliorer les vaccins, on pourrait arriver à élaborer des stratégies plus efficaces, plus cohérentes et plus respectueuses des appréhensions d'autrui au sujet de l'une des plus importantes avancées de santé publique du dernier millénaire. 


\section{BIBLIOGRAPHIE}

Betsch, C., F. Renkewitz, T. Betsch, and C. Ulshöfer. 2010. "The Influence of Vaccine- Critical Websites on Perceiving Vaccination Risks." Journal of Health Psychology 15 (3): 446-55. http://dx.doi. org/10.1177/1359105309353647.

Galdi, S., L. Arcuri, and B. Gawronski. 2008. "Automatic Mental Associations Predict Future Choices of Undecided Decision-Makers." Science 321 (5892): 1100-2. http://dx.doi.org/10.1126/science.1160769.

Goethe, Johann Wolfgang. 1774. The Sorrows of Young Werther. Leipzig: Weygand'sche Buchhandlung.

Gust, D.A., N. Darling, A. Kennedy, and B. Schwartz. 2008. "Parents with Doubts about Vaccines: Which Vaccines and Reasons Why." Pediatrics 122 (4): 718-25. http://dx.doi.org/10.1542/peds.2007-0538.

Jacobson, R.M., P.V. Targonski, and G.A. Poland. 2007. "A Taxonomy of Reasoning Flaws in the Anti-Vaccine Movement." Vaccine 25 (16): 3146-52. http://dx.doi.org/10.1016/j.vaccine.2007.01.046.

MacDonald, N.E., J. Smith, and M. Appleton. 2012. "Risk Perception, Risk Management and Safety Assessment: What Can Governments Do to Increase Public Confidence in Their Vaccine System?" Biologicals 40 (5): 384-8. http://dx.doi.org/10.1016/j.biologicals.2011.08.001.

Nan, X., and K. Madden. 2012. "HPV Vaccine Information in the Blogosphere: How Positive and Negative Blogs Influence Vaccine-Related Risk Perceptions, Attitudes, and Behavioral Intentions." Health Communication 27 (8): 829-36. http://dx.doi.org/10.1080/10410236.2012.661348.

Riphagen-Dalhuisen, J., G. Gefenaite, and E. Hak. 2012. "Predictors of Seasonal Influenza Vaccination among Healthcare Workers in Hospitals: A Descriptive Meta-Analysis." Occupational and Environmental Medicine 69 (4): 230-5. http://dx.doi.org/10.1136/oemed-2011-100134.

Salmon, D.A., L.H. Moulton, S.B. Omer, M.P. DeHart, S. Stokley, and N.A. Halsey. 2005. "Factors Associated with Refusal of Childhood Vaccines among Parents of School-Aged Children: A Case-Control Study." Archives of Pediatrics \&Adolescent Medicine 159 (5): 470-6. http://dx.doi.org/10.1001/archpedi.159.5.470.

Scullard, P., C. Peacock, and P. Davies. 2010. "Googling Children's Health: Reliability of Medical Advice on the Internet." Archives of Disease in Childhood 95 (8): 580-2. http://dx.doi.org/10.1136/adc.2009.168856. 